\title{
Hyperlactatemia and concurrent use of antiretroviral therapy among HIV infected patients in Uganda
}

\author{
*Waiswa $\mathrm{M}^{1}$, Byarugaba BB ${ }^{1}$, Ocama $\mathrm{P}^{1}$, Mayanja-Kizza $\mathrm{H}^{1}$, Seremba E ${ }^{1}$, Ganguli $\mathrm{S}^{2}$, Crowther $\mathrm{M}^{2}$ \\ Colebunders $\mathrm{R}^{3,4}$
}

1. Department of Medicine, Makerere University College of Health Sciences, Mulago Hospital, Kampala, Uganda

2. Faculty of Medicine, McMaster University College of Health Sciences, Ontario, Canada.

3. Epidemiology and Social Medicine, University of Antwerp.

4. Department of Clinical Sciences Institute of Tropical Medicine and University of Antwerp, Belgium

\begin{abstract}
Background: We determined the prevalence and factors associated with hyperlactatemia among HIV patients admitted on the emergency ward of a national hospital in Uganda.

Objective: We were specifically interested in knowing whether there was an association between clinically significant hyperlactatemia and concurrent antiretroviral therapy (ART) use.

Methods: A cross sectional descriptive study enrolled 303 HIV infected patients at a national referral hospital between March and April 2008. We consecutively recruited all eligible HIV infected patients above 18 years admitted on the emergency ward. Data were collected on socio-demographic, clinical and laboratory characteristics. Lactate levels were measured using the Accutrend ${ }^{\circledR}$ portable lactate analyser. Data analysis was performed using Stata 10.0; P-value of $<0.05$ was considered to be significant.

Results: Three hundred and three HIV infected patients were recruited. Prevalence of hyperlactatemia (lactate $\geq 2.5 \mathrm{mmol} /$ L) was $252(83.2 \%$ ). Clinically significant hyperlactatemia (lactate $\geq 4 \mathrm{mmol} / \mathrm{L}$ ) was present in $105 / 303(34.6 \%)$ patients. There was no association between use of ART and clinically significant hyperlactatemia. In the multivariate analysis, body weakness 1.91 (1.09-3.35), skin rash 3.18 (1.11-9.10) and tachypnoea 1.04 (1.01-1.07) were independently associated with clinically significant hyperlactatemia.

Conclusion: There was a high prevalence of clinically significant hyperlactatemia among HIV infected patients but it was not associated with concurrent antiretroviral use.

African Health Sciences 2012; 12(3):268 - 275 http://dx.doi.org/10.4314/ahs.v12i3.4
\end{abstract}

\section{Introduction}

Hyperlactatemia is the most common cause of metabolic acidosis in acutely ill patients. Lactic acidosis is considered to be present if the plasma lactate concentration exceeds $4-5 \mathrm{mmol} / \mathrm{L}$ even among patients without systemic manifestations of acidosis. The causes of lactic acidosis are divided into those associated with impaired tissue oxygenation (type A) as occurs in sepsis and shock states, and those in which a systemic impairment in tissue oxygenation is not apparent (type B) as occurs in mitochondrial dysfunction due to antiretroviral drug (ART) toxicity. ${ }^{1}$

\begin{tabular}{l}
\hline *Correspondence author: \\
Dr Musa Waiswa \\
Department of Medicine \\
Makerere University College of Health Sciences \\
Kampala, Uganda \\
P.O.Box 7072, Kampala, Uganda \\
Phone (256) 414541188 \\
Email: musa600@gmail.com \\
\hline
\end{tabular}

Since the introduction of potent antiretroviral drugs (ART), the incidence of opportunistic infections and deaths among HIV infected patients has dramatically declined. ${ }^{2,3}$ On the other hand, ART related toxicities such as hyperlactatemia have become a major concern in the management of HIV infected persons. ${ }^{4}$ In a mortality prediction study done in Uganda by Moore et al, lactate levels over $4 \mathrm{mmol} /$ L predicted a 7-fold higher mortality among patients with sepsis. In that study however, only10(13\%) patients of the study population were on ART and no association was found between ART use and hyperlactatemia. ${ }^{5}$ Prior to that study, Mwebaze et al in their retrospective chart review at the Adult Infectious Diseases Clinic in Uganda found a high prevalence of symptomatic hyperlactatemia (median serum lactate level of $6.6 \mathrm{mmol} / \mathrm{L}$ ) among patients on ART. ${ }^{6}$ Furthermore, et al in Tanzania reported a high mortality of $27.4 \%$ among children admitted with febrile illness and raised blood lactate levels although there was no mention of HIV status or

African Health Sciences Vol 12 No 3 September 2012 
ART use in this study. ${ }^{7}$ Severe sepsis is likely to be the predominant cause of lactic acidosis in most of these studies, but to know if ART use is associated with hyperlactatemia is a worthwhile question.

We therefore determined the prevalence of hyperlactatemia among HIV infected patients and associated factors including specifically ART use.

\section{Methods}

This was a cross sectional study conducted during the months of March and April 2008 at Mulago hospital in Kampala, Uganda. Mulago hospital is Uganda's national referral hospital and teaching hospital of Makerere University. This hospital admits over 12000 patients every year to the medical emergency ward. Patients are assessed and monitored over a 24 hour period at the medical emergency ward before being transferred to the subspecialty units.

The sample size was calculated using Kish and Leslie formula for cross- sectional studies with a p- value significance of $95 \%$ confidences giving a total of 288 patients. Considering a 10\% none response rate, a total of 303 participants were recruited. We consecutively recruited previously tested HIV infected patients (documented evidence of HIV positive result) admitted to the medical emergency ward as per the study protocol. Patients were included if they were HIV positive, age $>18$ years, and had given written informed consent to participate in the study. The study was approved by the institutional review board of the College of Health Sciences, Makerere University and the Uganda National Council of Science and Technology. We collected data on demographics, presenting symptoms and clinical examination findings, laboratory tests including renal function tests and serum bicarbonate levels and recorded all information on a pre-designed data collection tool.

A finger prick was used to obtain one drop of blood to determine blood lactate levels using the Accutrend portable lactate analyzer ${ }^{\circledR}$ (Sports Resource Group, USA) with sensitivity and specificity of $95.9 \%$ and $63.8 \%$ respectively. Further validation studies for the point-of-care lactate device by Moyo et al in Botswana found a strong correlation (Pearson correlation coefficient of $0.92(95 \%$ CI $0.88-0.95)$ between the portable device and conventional methods for blood lactate measurement. ${ }^{8,9}$ The use of point-of-care tests for lactic acid levels in an HIV treatment program in rural Haiti greatly assisted clinical decision-making with regard to patients on

African Health Sciences Vol 12 No 3 September 2012
ART who had symptoms suggestive of lactic $\operatorname{acidosis}^{10}$. This instrument uses enzymatic determination and reflectance photometry of lactate in the plasma portion of whole blood by use of a measurement strip. Results are available within 60 seconds of blood application. Five mLs of blood was drawn from the antecubital fossa for determining serum electrolytes and renal function tests (Cobas Integra machine, Roche, German) within 1-2 hours of collecting the blood samples. Data analysis was performed using Stata statistical software: Release 10. College station, TX: Stata Corp LP. The prevalence of hyperlactatemia was calculated as the proportion of patients with lactate levels of $\geq 2.5 \mathrm{mmol} / \mathrm{L}$ and clinically significant hyperlactatemia as lactate of $\geq 4 \mathrm{mmol} / \mathrm{L}$.

Bivariate analysis was done to assess the relationship between the occurrence of clinically significant hyperlactatemia and associated factors. Hyperlactatemia was categorized as present or absent using the clinically significant lactate cut off of $\geq 4 \mathrm{mmol} / \mathrm{L}$, a cut off value found to be predictive of a 7 -fold increase in mortality among patients with sepsis in a predominantly HIV infected population in Uganda. ${ }^{5}$ Association between predictor variables and clinically significant hyperlactatemia were established using Odds ratios, p-values and 95\% confidence intervals. A $\mathrm{p}$-value of $\leq 0.05$ was considered to be statistically significant. Statistically significant factors at bivariate analysis were entered into a logistic regression model for multivariate analysis to determine the factors that were independently associated with clinically significant hyperlactatemia. To further evaluate the association between ART use and hyperlactatemia, the student's t-test was used.

\section{Results}

\section{Background characteristics}

Five hundred and two patients were screened for eligibility into the study but $303(60.4 \%)$ met the inclusion criteria for this analysis as shown in figure 1. Table 1 shows the demographic and clinical characteristics of the study participants. One hundred fifty five $(51.2 \%)$ of them were males. The median age of study participants was 35 years (range 17 to 82 years). The majority of patients presented with a subjective fever totalling 186(61.4\%) and cough 155(51.2\%) but only $106(35 \%)$ had a temperature $\geq 37.5^{\circ} \mathrm{C}$ at the time of recruitment. One hundred fifty $(49 \%)$ of the participants had a tachypnoea of $>20$ breath/minute, and $138(45.5 \%)$ had a tachycardia of $>90$ beats/minute. 
Figure 1: Profile of study participants

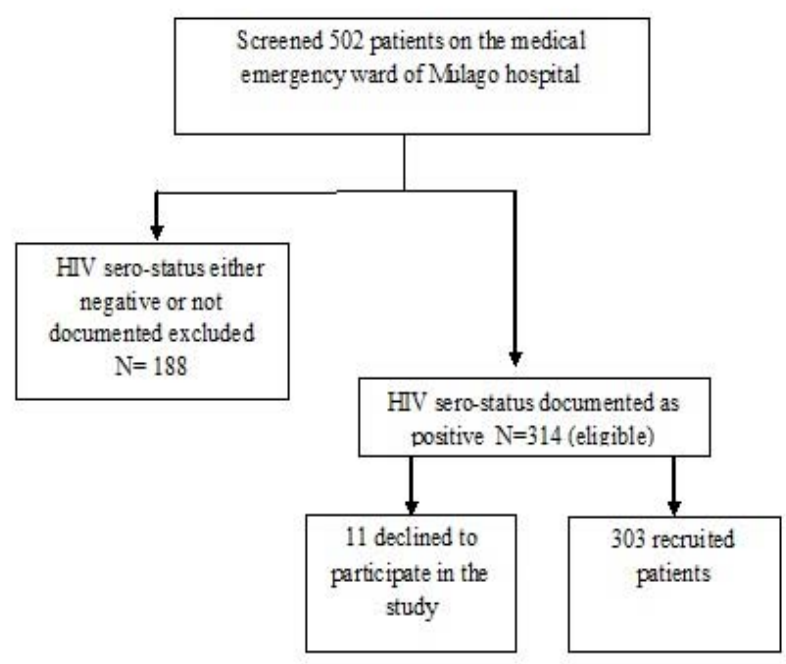

Table 1: Socio-demographic and clinical characteristics of study participants

\begin{tabular}{|c|c|c|}
\hline Socio-demographic characteristics & Frequency $(\mathrm{N}=303)$ & Percentage $(\%)$ \\
\hline Male gender & 155 & 51.2 \\
\hline Mean age (years) $36 \pm 10$, Median $35(17-82)$ & & \\
\hline \multicolumn{3}{|l|}{ Alcohol and drug use } \\
\hline History of alcohol use & 71 & 23.4 \\
\hline \multicolumn{3}{|l|}{ Clinical characteristics Systemic symptoms } \\
\hline Fever & 186 & 61.4 \\
\hline Body weakness & 87 & 28.7 \\
\hline \multicolumn{3}{|l|}{ Respiratory symptoms } \\
\hline Dyspnoea & 59 & 19.5 \\
\hline Cough & 155 & 51.2 \\
\hline \multicolumn{3}{|l|}{ Gastrointestinal symptoms } \\
\hline Abdominal pain & 70 & 23.1 \\
\hline Diarrhoea & 52 & 17.2 \\
\hline Vomiting & 82 & 27.1 \\
\hline \multicolumn{3}{|l|}{ CNS symptoms } \\
\hline Confusion & 26 & 8.6 \\
\hline Dizziness & 18 & 5.90 \\
\hline Temperature $\left(\mathrm{T} \geq 37.5^{\circ} \mathrm{C}\right)$ & 106 & 35 \\
\hline Respiratory rate $(\bar{R} R>20$ breaths per minute) & 150 & 49 \\
\hline Heart rate (HR $>90$ beats per minute) & 138 & 45.5 \\
\hline Skin rash & 18 & 6.0 \\
\hline \multicolumn{3}{|l|}{ WHO clinical stage of HIV/AIDS } \\
\hline Stage 1 & 00 & 00.0 \\
\hline 2 & 39 & 12.8 \\
\hline 3 & 172 & 56.7 \\
\hline 4 & 92 & 30.4 \\
\hline
\end{tabular}

Mean systolic blood pressure $100 \pm 14$ 
The mean systolic blood pressure was $100 \pm 14 \mathrm{mmHg}$. Two hundred sixty four $(87.1 \%)$ of the study subjects had WHO HIV stage 3 and 4 disease. Table 2 shows the patients who were on ART. A total of $95(31 \%)$ participants were on ART. The most frequently prescribed ART combinations were zidovudine/lamivudine and stavudine/ lamivudine constituting $60 \%$ and $23 \%$ respectively.

Table 2: ARV use among study participants $(\mathrm{N}=303)$

\begin{tabular}{|c|c|c|}
\hline Types of ARVs & Frequency $(\mathrm{N}=95)^{*}$ & $(\%)$ \\
\hline Lamivudine & 80 & 82.2 \\
\hline Zidovudine & 57 & 60.0 \\
\hline Nevirapine & 46 & 48.4 \\
\hline Efavirenz & 38 & 40.0 \\
\hline Stavudine & 22 & 23.2 \\
\hline $\begin{array}{l}\text { Tenofovir/Emtric } 1 \\
\text { itabine }\end{array}$ & 15 & 15.8 \\
\hline Lopinavir/Ritonavir & r 2 & 2.1 \\
\hline $\begin{array}{l}\text { Zidovudine/Lami } 5 \\
\text { vudine }\end{array}$ & 57 & 60.0 \\
\hline $\begin{array}{l}\text { Stavudine/ Lamivu } 2 \\
\text { dine }\end{array}$ & & 23.2 \\
\hline
\end{tabular}

The overall prevalence of hyperlactatemia was $83.2 \%$ and the prevalence of clinically significant hyperlactatemia was $34.6 \%$. Table 3 shows association between demographic and clinical characteristics with clinically significant hyperlactatemia. We analysed individual symptoms and their association with clinically significant hyperlactatemia and subsequently grouped them into systems. Dyspnoea, body weakness, respiratory symptoms and skin rash were significantly associated with clinically significant hyperlactatemia with $\mathrm{p}$ values of $0.02,0.01,0.03$ and 0.02 respectively. There was no association between use of ART and clinically significant hyperlactatemia $(\mathrm{p}=0.11)$. Further analysis using a t-test, confirmed no association between ART use and hyperlactatemia with mean lactate levels of 3.92 (CI 3.60-4.24) and 3.62 (CI 3.44-3.80) among patients on ART and ART naive, respectively $(\mathrm{p}=0.08)$. The mean duration of ART use was $8.5 \pm 10.2$ months with a median of 4 months (range 2-12) and was not associated with clinically significant hyperlactatemia.

Table 3: Association between demographic and clinical characteristics with clinically significant hyperlactatemia

\begin{tabular}{|c|c|c|c|c|c|}
\hline Predictor variables & & $\begin{array}{l}\text { Lactate } \\
<4 \mathrm{mmol} / \mathrm{L} \\
\mathrm{N}=198\end{array}$ & $\begin{array}{l}\text { Lactate } \\
\geq 4 \mathrm{mmol} / \mathrm{L} \\
\mathrm{N}=105\end{array}$ & $\begin{array}{l}\text { Odds Ratio } \\
\text { (confidence interval) }\end{array}$ & p-value \\
\hline \multicolumn{6}{|l|}{ Socio demographic } \\
\hline \multicolumn{2}{|l|}{ Gender Male } & $96(64.0)$ & $52(35.0)$ & & \\
\hline \multicolumn{2}{|l|}{ Female } & $102(65.8)$ & $53(34.2)$ & $0.96(0.59-1.54)$ & 0.86 \\
\hline \multicolumn{2}{|l|}{ Age $(<40)$} & $131(63.6)$ & $75(36.4)$ & & \\
\hline \multicolumn{2}{|l|}{$(\geq 40)$} & $67(69.1)$ & $30(30.9)$ & $0.35(0.46-1.31)$ & 0.35 \\
\hline \multicolumn{6}{|l|}{ Symptoms } \\
\hline \multirow[t]{2}{*}{ Dyspnoea } & No & $167(68.4)$ & $77(31.2)$ & & \\
\hline & Yes & $31(52.5)$ & $28(47.5)$ & $1.95(1.09-3.51)$ & 0.02 \\
\hline \multirow[t]{2}{*}{ Cough } & No & $103(69.6)$ & $45(30.4)$ & & \\
\hline & Yes & $95(61.3)$ & $60(38.7)$ & $1.44(0.89-2.3)$ & 0.13 \\
\hline \multirow[t]{2}{*}{ Vomiting } & No & $146(66.0)$ & $75(34.0)$ & & \\
\hline & Yes & $52(63.4)$ & $30(36.6)$ & $1.12(0.66-1.91)$ & 0.66 \\
\hline \multirow[t]{2}{*}{ Body weakness } & No & $151(69.9)$ & $65(30.1)$ & & \\
\hline & Yes & $47(54.0)$ & $40(45.9)$ & $1.98(1.18-3.32)$ & 0.01 \\
\hline \multirow[t]{2}{*}{ Fever } & No & $69(58.9)$ & $48(41.0)$ & & \\
\hline & Yes & $129(69.4)$ & $57(30.6)$ & $0.64(0.39-1.03)$ & 0.07 \\
\hline \multirow[t]{2}{*}{ Systemic symptoms } & No & $45(60.8)$ & $29(39.2)$ & & \\
\hline & Yes & $153(66.8)$ & $76(33.2)$ & $0.77(0.45-1.33)$ & 0.35 \\
\hline
\end{tabular}


Continuation of table 3

\begin{tabular}{|c|c|c|c|c|c|}
\hline Predictor variables & & $\begin{array}{l}\text { Lactate } \\
<4 \mathrm{mmol} / \mathrm{L} \\
\mathrm{N}=198\end{array}$ & $\begin{array}{l}\text { Lactate } \\
\geq 4 \mathrm{mmol} / \mathrm{L} \\
\mathrm{N}=105\end{array}$ & $\begin{array}{l}\text { Odds Ratio } \\
\text { (confidence interval) }\end{array}$ & p-value \\
\hline \multirow[t]{2}{*}{ Respiratory } & $\mathrm{No}$ & $90(72.6)$ & $34(27.4)$ & & \\
\hline & Yes & $108(60.3)$ & $71(39.7)$ & $1.74(1.06-2.87)$ & 0.03 \\
\hline \multirow{2}{*}{ Gastrointestinal } & No & $107(67.3)$ & $52(32.7)$ & & \\
\hline & Yes & $91(63.2)$ & $53(36.8)$ & $1.19(0.75-1.93)$ & 0.45 \\
\hline \multirow[t]{2}{*}{ CNS symptoms } & No & $171(65.8)$ & $89(34.2)$ & & \\
\hline & Yes & $27(62.3)$ & $16(37.2)$ & $1.14(0.58-2.23)$ & 0.70 \\
\hline \multirow[t]{2}{*}{ Skin rash } & No & $191(67.0)$ & $94(33.0)$ & & \\
\hline & Yes & $7(38.9)$ & $11(61.0)$ & $3.19(1.18-8.56)$ & 0.02 \\
\hline \multirow[t]{2}{*}{ WHO clinical stage } & 2 & $24(62.0)$ & $15(38.0)$ & & \\
\hline & $3 \& 4$ & $173(66.0)$ & $91(34.0)$ & $0.89(0.43-1.81)$ & 0.75 \\
\hline \multirow[t]{2}{*}{ Anti-retroviral drugs } & No & $142(68.3)$ & $66(31.7)$ & & \\
\hline & Yes & $56(58.9)$ & $39(41.1)$ & $1.50(0.90-2.48)$ & 0.11 \\
\hline
\end{tabular}

Table 4(a) shows association between physical signs and laboratory findings with clinically significant hyperlactatemia. Only tachypnoea was associated with clinically significant hyperlactatemia $(\mathrm{p}<0.01)$. There was no other systemic inflammatory response syndrome (SIRS) criteria associated with clinically significant hyperlactatemia. Laboratory findings confirmed presence of acidosis, as shown by a significantly high anion gap and low bicarbonate levels, among patients with clinically significant hyperlactatemia with p-values of 0.03 and 0.05 respectively. Table 4(b) shows factors independently associated with clinically significant hyperlactatemia among study participants in the multivariate analysis. Only body weakness $(p=0.02)$, tachypnoea $(p<0.01)$ and skin rash $(\mathrm{p}=0.03)$ were independently associated with clinically significant hyperlactatemia.

Table 4a: Association between physical signs and laboratory characteristics with clinically significant hyperlactatemia (lactate $\geq 4 \mathrm{mmol} / \mathrm{L}$ )

\begin{tabular}{llll}
\hline Variable (mean) & $\begin{array}{l}\text { Lactate } \\
<4 \mathbf{m m o l} / \mathbf{L} \\
\mathbf{N = 1 9 8}\end{array}$ & $\begin{array}{l}\text { Lactate } \\
\mathbf{2} \text { (mmol/L } \\
\mathbf{N = 1 0 5}\end{array}$ & p-value \\
\hline Clinical signs & & & \\
Temperature $\left({ }^{\circ} \mathrm{C}\right)$ & 37.2 & 37.1 & 0.45 \\
Respiratory rate $(\mathrm{b} / \mathrm{min})$ & 22 & 27 & $<\mathbf{0 . 0 1}$ \\
Heart rate (beats/min) & 90 & 94 & 0.06 \\
Blood pressure $(\mathrm{mmHg})$ & 101 & 98 & 0.10 \\
Laboratory parameters & & & \\
$\mathrm{Na}^{+}(\mathrm{mmol} / \mathrm{L})$ & 129 & 131 & $\mathbf{0 . 0 5}$ \\
$\mathrm{K}^{+}(\mathrm{mmol} / \mathrm{L})$ & 4.4 & 4.3 & 0.40 \\
$\mathrm{Cl}^{-}(\mathrm{mmol} / \mathrm{L})$ & 93 & 94 & 0.08 \\
$\mathrm{HCO}_{3}^{-}(\mathrm{mmol} / \mathrm{L})$ & 18.4 & 17.7 & $\mathbf{0 . 0 5}$ \\
$\mathrm{BUN}(\mathrm{mg} / \mathrm{dl})$ & 32 & 33 & 0.77 \\
$\mathrm{Creatinine}(\mathrm{mg} / \mathrm{dl})$ & 1.2 & 1.15 & 0.17 \\
Anion gap $(\mathrm{mmol} / \mathrm{L})$ & 17 & 19 & $\mathbf{0 . 0 3}$ \\
\hline
\end{tabular}

SIRS criteria: Fever $>38^{\circ} \mathrm{C}$, or $<36^{\circ} \mathrm{C}$, Heart rate $>90$ beats per minute, Respiratory rate $>20$ breaths per minute. 
Table 4b: Factors associated with clinically significant hyperlactatemia in the multivariate analysis

\begin{tabular}{lll}
\hline Variables & $\begin{array}{l}\text { Odds Ratio } \\
\text { (confidence } \\
\text { interval) }\end{array}$ & P-value \\
\hline Dyspnoea & $1.82(0.93-3.54)$ & 0.08 \\
Weakness & $1.91(1.09-3.35)$ & $\mathbf{0 . 0 2}$ \\
Skin rash & $3.18(1.11-9.10)$ & $\mathbf{0 . 0 3}$ \\
Respiratory rate (breaths/min) & $1.04(1.01-1.07)$ & $<\mathbf{0 . 0 1}$ \\
Heart rate (beats/min) & $1.14(0.65-2.01)$ & 0.65 \\
\hline
\end{tabular}

\section{Discussion}

This study was undertaken to determine the prevalence of hyperlactatemia among HIV infected patients and associated factors including particularly ART use. We demonstrated a high prevalence of hyperlactatemia among HIV infected patients admitted to our medical emergency ward that was not associated with concurrent ART use. The overall prevalence of hyperlactatemia as defined by blood lactate levels of $\geq 2.5 \mathrm{mmol} / \mathrm{L}$ in our study was $83.2 \%$. This prevalence is higher than that reported by Mina et al of $65 \%$ in their prospective longitudinal study of chronic hyperlactatemia among 349 HIV infected out patients on ART in Australia. ${ }^{11}$ Likewise it is higher than findings from an earlier prospective study in Uganda that revealed an overall prevalence of hyperlactatemia of $68.6 \%$ among patients with sepsis in a predominantly HIV infected population. ${ }^{5}$ The hyperlactatemia prevalence in our study is much higher than the $24.8 \%$ reported by Marceau et al in their prospective cross-sectional study of the frequency, risk factors and outcome of hyperlactatemia among $282 \mathrm{HIV}$ positive out patients in France. ${ }^{12}$ The difference in the prevalence may be as result of the study population where patients in our study were acutely ill and hospitalized, compared to out patients in studies done in France and Australia, suggesting a possible role of sepsis in causation.

The prevalence of clinically significant hyperlactatemia among our study participants was $34.6 \%$. This result is similar to the findings by Moore et al who found that up to $33.5 \%$ of patients admitted at Mulago hospital with sepsis had blood lactate levels of $\geq 4 \mathrm{mmol} / \mathrm{L}$, a value found to be predictive of a 7 -fold increase in mortality (with an $81 \%$ accuracy). This value is similar to that reported in a randomized controlled trial of HIV infected women on ART in Botswana in which $31 \%$ of the patients had hyperlactatemia of $\geq 4.40 \mathrm{mmol} / \mathrm{L} .{ }^{5,13}$ The diversity in the prevalence of hyperlactatemia in developing and the developed countries as shown above, is probably the result of differences in the geographical distribution of underlying opportunistic infections that appear to drive hyperlactatemia in HIV infected patients. Indeed HIV infection with high prevalence in sub-Saharan Africa has been shown to be independently associated with bacteremia. In an earlier prospective study of community-acquired blood stream infections among 299 febrile adult medical admissions in Uganda, the overall prevalence of bacteremia was $24 \%$ and was significantly higher among HIV-infected patients. ${ }^{14,15}$ Infections can induce a systemic inflammatory response syndrome(SIRS) that can progress to cardiovascular compromise leading to global tissue hypoxia, and elevations in blood lactate from anaerobic cellular metabolism.

In a recent prospective observational study of hypoglycaemia related in-hospital mortality among Ugandan patients with severe sepsis, the prevalence of hyperlactatemia (blood lactate $>$ $4 \mathrm{mmol} / \mathrm{L}$ ) was $37.7 \%$. This value is slightly higher than our finding partly because the study participants were enrolled if they met the SIRS criteria, suggesting sepsis as compared to our study that enrolled all patients requiring hospital admission for various reasons. Blood lactate of $>4 \mathrm{mmol} / \mathrm{L}$ significantly predicted in-hospital mortality. In a related study of aggregate evaluable organ dysfunction as a predictor of in-hospital mortality from sepsis, blood lactate of $>4 \mathrm{mmol} / \mathrm{L}$ was among the laboratory predictors of mortality. ${ }^{16,17}$ In both of these studies however, the contribution of concurrent ART use to hyperlactatemia and mortality was not well documented creating need for its evaluation in our study.

Respiratory symptoms, unlike fever, were significantly associated with clinically significant 
hyperlactatemia. The findings are similar to reports by Marceau et al in France, who found that respiratory symptoms, unlike fever were significantly associated with hyperlactatemia at univariate analysis. ${ }^{12}$ Such an observation raises the possibility of respiratory compensation for the metabolic acidosis, especially in the absence of fever. It is, however, still possible that underlying respiratory pathology was driving the hyperlactatemia. The lack of a septic screen and diagnoses for our study patients precluded us from making any authentic statements about the "chicken and the egg." Furthermore skin rash was independently associated with clinically significant hyperlactatemia and could be a reflection of a septic focus for bacteraemia and consequent septicaemia that is a well documented cause of hyperlactatemia.

As shown in table $295(31 \%)$ of the study patients were taking anti-retroviral medications. Use of these drugs was not associated with clinically significant hyperlactatemia. This finding is similar to reports by Bonnet et al in their case-controlled study of risk factors for lactic acidosis among patients on ART in which no association was found between cumulative exposure to ART (nucleoside reversetranscriptase inhibitors) and increased risk of lactic acidosis ${ }^{18}$. Lack of association between hyperlactatemia and exposure to ART, was also reported by Moore et al in their mortality prediction study of severe sepsis in a predominantly HIV infected population in Uganda, although only 10(13.9\%) patients were on ART ${ }^{5}$. Our study findings showed that concurrent use of ART among hospitalized HIV-infected patients is not associated with clinically significant hyperlactatemia and hence the need to look for other causes of hyperlactatemia in this population. Well controlled prospective studies are required to establish causes of hyperlactatemia among HIV infected patients requiring hospital admission.

Our study had some limitations. We were unable to conduct a septic screen or document specific patient diagnoses since the study was conducted on the emergency ward where patients are admitted for only 24 hours, before being transferred to the appropriate sub-speciality medical units, for further work up and management. Furthermore, the white blood cell component of the SIRS criteria was not done because this service was not routinely available on this ward.

\section{Conclusion}

Clinically significant hyperlactatemia is common among HIV infected patients admitted on the emergency ward of Mulago hospital in Uganda and is not associated with concurrent ART use. There is need to explore other potential causes of hyperlactatemia in acutely ill patients before stopping antiretroviral drugs upfront. The study findings have important implications both for research and clinical practice. Future studies should prospectively describe the causes and factors associated with hyperlactatemia among acutely ill patients and clinicians should have a high index of suspicion for hyperlactatemia and lactic acidosis syndromes among acutely ill patients.

\section{Acknowledgements}

The study was co-funded by the Flemish Interuniversity council (VLIR-UOS).

Sincere thanks go to S. Reynolds for the logistical support including the provision of the lactate machine and strips. In addition, we are especially grateful to over research assistants including Drs. W. Sekabira, J. Kazibwe and R. Kakaire for dedication and hard work.

Conflict of Interest: None declared.

\section{References}

1. Luft C.F. Lactic Acidosis Update for Critical Care Clinicians. J Am Soc Nephrol 2001; 12:S1519.

2. Hung CC, Chen MY, Hsieh SM, Sheng WH, Chang SC. Clinical spectrum, morbidity and mortality of AIDS in Taiwan. A 5-year study. JAIDS $2000 ; 24: 378-385$.

3. Palella F, Drlaney M, Moorman C, et al: Declining morbidity and mortality among patients with advanced HIV infection. N Eng J Med 1998; 338:853-860.

4. Fellay J, Boubaker K, Ledergerber B, et al: Prevalence of adverse events associated with potent antiretroviral treatment: Swiss HIV cohort study. Lancet 2001; 358:1322-1327.

5. Moore. C, Jacob S, Pinkerton R, et al Point of care lactate testing predicts mortality of severe sepsis in a predominantly HIV type I- infected patient population in Uganda. Clin Infect Dis. 2008 Jan 15; 46(2):215-22.

6. Mwebaze S P, Castelnuovo B, Birabwa E M, Ocama P, Kambugu A. Symptomatic hyperlactatemia associated with nucleoside reverse-transcriptase inhibitor use in HIVinfected patients. A report of 24 cases in a 
resource-limited setting (Uganda). Clin Infect Dis 2007

7. Mtove G, Nadjm B,Hendriksen C.E et al. Pointof-Care measurement of blood lactate in children admitted with febrile illness to an African district hospital CID 2011; 53:548-554.

8. Perez HE, Dawood H, Chetty U, Esterhuizen T, Bizaare M. Validation of the Accutrend lactate meter for hyperlactatemia screening during antiretroviral therapy in a resource-poor setting; International Journal of infectious diseases 2008;533:14

9. Moyo S, Bussmann H, Mangwendeza P et al. Validation of a point -of-care lactate device for screening at-risk adults receiving combination antiretroviral therapy in Botswana. J Antivir Antiretrovir. 2011,3:4

10. Ivers LC, Mukherjee JS. Point of care testing for antiretroviral therapy-related lactic acidosis in resource-poor settings. AIDS 2006; 20:779-780

11. Mina J,Moore CB,James $\mathrm{R}$ et al. Chronic hyperlactatemia in HIV-infected patients taking antiretroviral therapy. AIDS 2001; 15:717-723.

12. Marceau G, Sapin V, Jacomet $C$ et al. Frequency, risk factors, and outcome of hyperlactatemia in HIV-positive persons: Implications for management of treated patients. Clinical Chemistry 2003;49:1154-1162
13. Wester CW, Okechukwu AO, MUIR AT et al Higher-Than-Expected rates of lactic acidosis among highly active antiretroviral therapy-treated women in Botswana. J AIDS 2007; 46:318-322.

14. Berkley JA, Lowe BS, Mwangi I, et al. Bacteremia among children admitted to a rural hospital in Kenya. N Engl J Med 2005; 352:39-47.

15. Ssali FN, Kamya MR, Wabwire-Mangen F, et al. A prospective study of community-acquired bloodstream infections among febrile adults admitted to Mulago Hospital in Kampala, Uganda. J Acquir Immune Defic Syndr Hum Retrovirol 1998;19:484-9

16. Ssekitoleko R, Jacob S, Banura P, et al. Hypoglycemia at admission is associated with in hospital mortality in Ugandan patients with severe sepsis. Crit Care Med 2011;10:2271-76

17. Ssekitoleko R,Pinkerton R,Muhindo R, Bhagani S, Moore C. Aggregate evaluable organ dysfunction predicts in-hospital mortality from sepsis in Uganda.

18. Bonnet F, Bonarek M,Morlat P et al. Risk factors for lactic acidosis in $\mathrm{HIV}$-infected patients treated with nucleoside reverse-transcriptase inhibitors: A case-control study. CID 2003; 36:1324-8. 